\title{
ULTRASONOGRAPHY OF BORDERLINE AND ASYMMETRIC INFANT HIPS - A CHALLENGE IN THE MANAGEMENT OF DEVELOPMENTAL DYSPLASIA OF THE HIP
}

\author{
DAN VASILESCU ${ }^{1}$, DAN COSMA ${ }^{2}$, DANA ELENA VASILESCU ${ }^{2}$, \\ CAROLINA MARIA SOLOMON ${ }^{1}$, ANDREI CORBU², \\ SORIN MARIAN DUDEA ${ }^{1}$
}

\author{
${ }^{1}$ Radiology Department, Iuliu Hatieganu University of Medicine and Pharmacy, \\ Cluj-Napoca, Romania \\ ${ }^{2}$ Pediatric Orthopedic Surgery Department, Iuliu Hatieganu University of \\ Medicine and Pharmacy, Cluj-Napoca, Romania
}

\section{Abstract}

Background and aim. Developmental dysplasia of the hip (DDH) is a potentially disabling disease. There are many diagnostic approaches, Graf method ultrasonography being the most popular imaging method. Although considered as a healthy condition, the existence of hips at the 60 degree limit or the asymmetries higher than 4 degrees between left and right side may be a source of unfavorable evolution and consequently of late diagnosed dysplasia cases.

Methods. The retrospective study was conducted in the Radiology Department of the Emergency Clinical County Hospital Cluj-Napoca, by retrospective analysis of the database containing 3013 records of the subjects presented for DDH assessment between January 2008 and December 2014. The study focuses on investigating two possible sources of missed cases by clinical-ultrasound management of DDH: borderline and asymmetric hips. Two conditions were studied in patients considered healthy according to Graf method: borderline hips (those with a angle value of $60^{\circ}$ and $61^{\circ}$ ) and asymmetric hips (left to right difference between the $\alpha$ angle values exceeds $\left.4^{\circ}\right)$. Three study groups were formed: healthy subjects, asymmetric/borderline subjects and patients with immature or mild dysplasia. The incidence of risk factors, clinical suspicion and the success of therapy were evaluated.

Results. There were no significant differences between the three groups regarding the role of the risk factors in DDH pathogenesis. Data reveal a high suspicion rate after the clinical examination, in groups II and III, compared to the healthy population. This means that from this point of view, Group II might be considered having at least a dysplastic prognosis. An increased correlation in the therapeutic results was observed between Group II patients and those from mild delayed maturation subgroups from Group III (IIa-, IIa+).

Conclusions. Asymmetric and borderline hips should be approached similarly to immature hips, clinical suspicion and the therapeutic outcome being similar.

Keywords: DDH, ultrasound Graf method, borderline hips, asymmetric hips, infant

\section{Background and aim}

Developmental dysplasia of the hip (DDH) is a condition with invalidity potential.

The diagnosis is based on the identification of risk factors, clinical examination and the appropriate use of imaging

Manuscript received: 24.04.2018

Received in revised form: 19.06.2018

Accepted: 02.07.2018

Address for correspondence: danvasi76@yahoo.com methods according to the age of the patient: hip ultrasound and classic pelvic radiography. The diagnostic method and complexity still remain in the international debate. It is widely accepted that for optimal therapeutic results the early diagnosis must be formulated until the age of 6 weeks $[1,2]$.

Although subjective, clinical examination is somewhat standardized in terms of tests performed: Ortolani, Barlow, assessment of abduction. Clinical examination 
results are influenced by many factors: patient age, the examiner's experience, degree of muscle tone, the status of the opposite hip etc [3].

The use of ultrasound as first intention imaging method is universally accepted. However, there is still a debate regarding the optimal time when it should be performed and the target group. Examination protocols differ according to the country or center. Two concepts are most widespread: 1) hip ultrasound only in patients with present risk factors and / or those with a clinical suspicion; 2) hip ultrasound as screening method for DDH, independent from clinical examination $[4,5,6]$.

Graf method is the most common and standardized ultrasound technique. It is based on a standard image that must meet several criteria of correctness. This image serves to determine $\alpha$ and $\beta$ angles, and it represents bone and cartilage coverage respectively of the femoral head. Although widely used, Graf method has contesters. They report an increased rate of positive diagnosis, but also late diagnosed DDH cases by radiography in patients considered healthy on the first ultrasound examination $[5,7,8,9]$.

Empirical observations of our group revealed that borderline and asymmetric hips might have unfavorable outcome.

Although asymmetric and border hips are not a rare discovery in ultrasound practice, literature is deficient in information.

The purpose of the paper is to assess the degree of risk of the evolution of these hips.

\section{Methods}

The study focuses on investigating two possible sources of missed cases by clinical-ultrasound management of DDH: borderline and asymmetric hips.

Borderline hips are those with $\alpha$ angle value of $60^{\circ}$ and $61^{\circ}$, in patients considered healthy according to Graf method.

Asymmetric hips in a patient are considered when the values of $\alpha$ angle are greater than $60^{\circ}$, but left to right difference between the $\alpha$ angle values exceeds $4^{\circ}$.

The study was conducted in the Radiology Department of the Emergency Clinical County Hospital Cluj-Napoca, by retrospective analysis of the database containing 3013 reports of the subjects presented for DDH assessment between January 2008 and December 2014. In all cases, hip ultrasound was performed according to the Graf method using Hitachi 8500 EUB US equipment with a 6.5-13 MHz linear transducer. A single operator having two years experience in hip ultrasound at the date of the onset of the study period performed all the examinations.

Due to the retrospective nature of the study the organization ethics committee waived informed consent.

Inclusion criteria consisted of: subjects presented for the first time to hip ultrasound with or without prior clinical examination and for whom examination data and ultrasound images were available.
Exclusion criteria were: severe dysplastic hips (IIc, IIIa, IIIb and IV), patients for whom either report data or images were missing.

Of the initial 3013 reports analyzed, after applying the above criteria, data for 2517 subjects represented the study group.

The age of the patients included in the study ranged from 2 weeks to 6 months (26 weeks), with a mean age value of 10.7 weeks.

Three independent groups were formed according to $\alpha$ angle values:

Group I included 1985 subjects considered healthy, with bilateral $\alpha>60^{\circ}$ and without a difference greater then $4^{\circ}$ between the hips $\alpha$ angle values.

Group II included 250 patients with borderline and/ or asymmetric hips as defined above.

Group III included 282 patients having at least one hip classified as DDH or immature (IIa-, IIa+ and IIb) according to Graf method.

On all patients of groups II and III, abduction devices were used according to the orthopedic surgeon decision. The same ultrasound operator using the same Graf method performed at follow-up examinations after 4-6 weeks of treatment for most of the patients of Group II and III.

Patients with follow-up were considered for comparing the outcome of groups II and III.

A favorable evolution was considered when an improvement of $\alpha$ angle values was discovered and at the end of the follow-up period, the patient was considered healthy complying with the following criteria: no borderline values or no asymmetry (Figure 1,2).
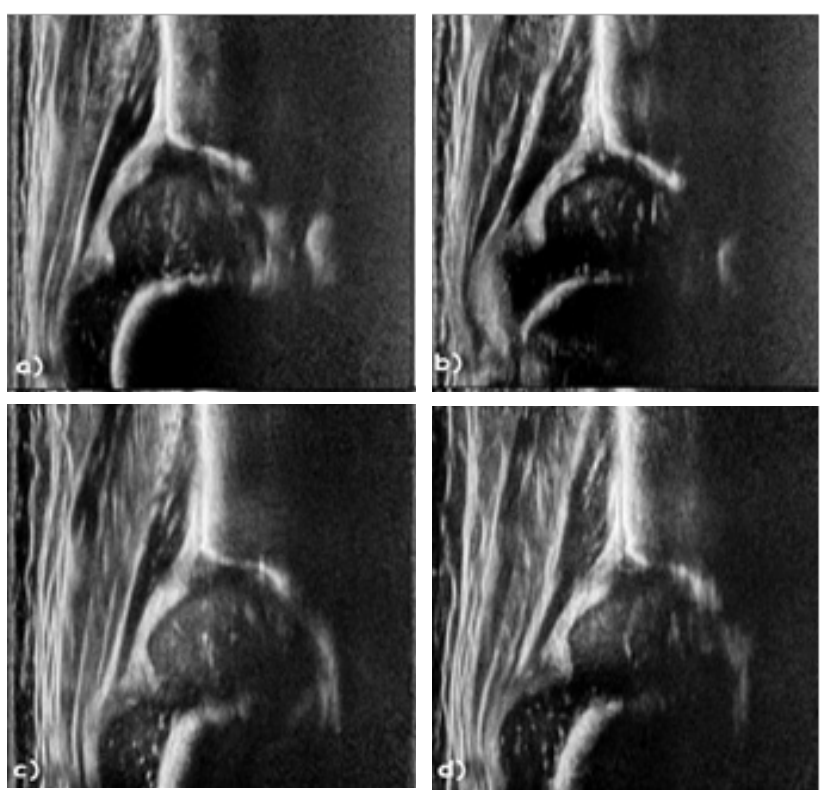

Figure 1. Asymmetric hips in evolution. a) and b) show the initial stage at the age of 5 weeks $-8^{\circ}$ difference between right and left hips alpha angle values (alpha angle measured for right and left hip were $71^{\circ}$, respectively $\left.63^{\circ}\right)$. c) and d) present the evolution after 5 weeks of abduction attitude (use of 2 diapers), $3^{\circ}$ difference $\left(72^{\circ}\right.$, respectively $\left.69^{\circ}\right)$, it shows an improved alpha angle on the left hip (from $63^{\circ}$ to $69^{\circ}$ ). 


\section{Medical Imaging}
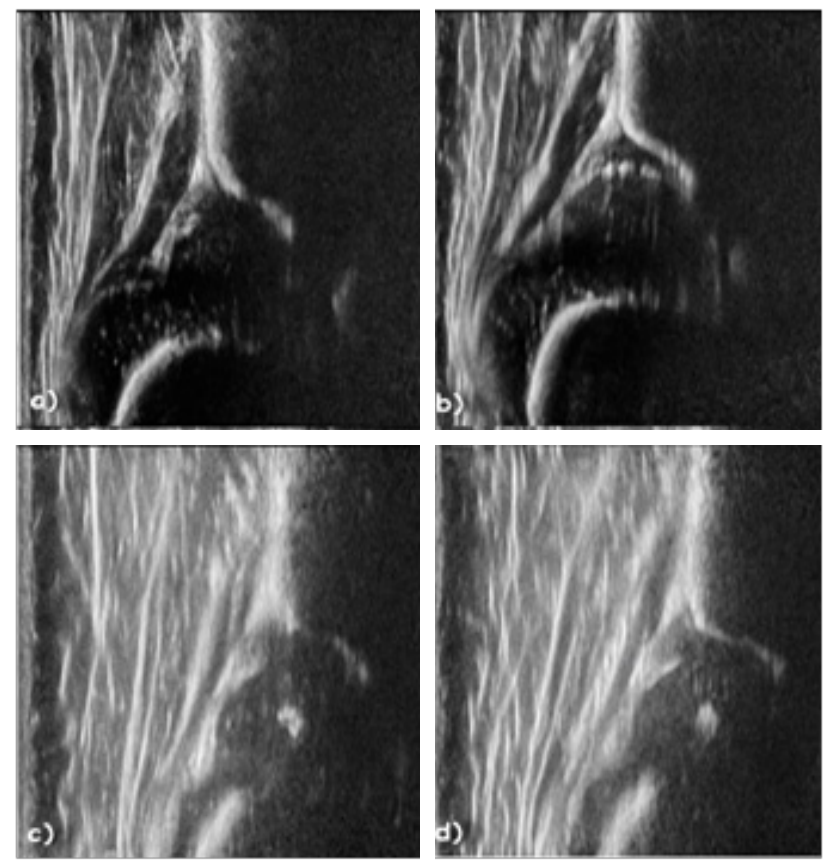

Figure 2. Bilateral borderline hips. a) and b) represent the initial stage at the age of 6 weeks. Both hips had an alpha angle value of $60^{\circ}$. c) and d) after 6 weeks of abduction treatment attitude it was observed an improvement by $3^{\circ}$ and respectively $4^{\circ}$ on the alpha angle values. Appearance of the ossification nucleus in both femoral heads represents an indirect sign of positive evolution.

Unfavorable evolution is defined as: halt or involution at a stage considered dysplastic (IIb or worse), obtaining or maintaining the asymmetry or a hip as border type (Figure 3).
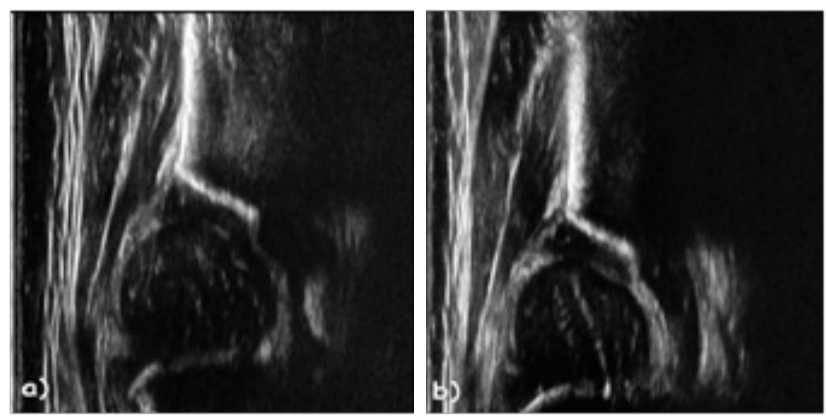

Figure 3. a) Borderline $60^{\circ}$ alpha angle left hip, b) stationary evolution at $61^{\circ}$, after 6 weeks of abduction treatment attitude, 2 diapers.

In order to find any correlation between the new supposed abnormal condition (asymmetric and borderline hips) and the known pathological conditions (immature and dysplastic hips), the incidence of risk factors (breech delivery, prematurity, family DDH history, twin pregnancy and associated musculoskeletal disorders as: club foot, torticollis, metatarsus adductus), clinical suspicion and the success of therapy were evaluated (Table I, II).
Statistical software used was Social Science Statistics with free access on http://www.socscistatistics.com. The Z-test was used, as recommended test to compare the population group's proportions and to assess the possible correlation between the same conditions presented in two different groups. $\mathrm{P}$ was considered statistically significant at a $\mathrm{p}$ value $<0.05$.

\section{Results}

The incidence of risk factor for DDH in Group I was $19.24 \%$ (382 patients). From the total 1985 patients, 1231 were clinically examined first. After the clinical examination, in 512 from these patients $(41.5 \%)$ a clinical suspicion of abnormal hip was formed.

In Group II the incidence o risk factors was $16.8 \%$ (42 patients). 179 patients from the Group II performed initially clinical examination and indication for ultrasound evaluation was formulated in $55.3 \%$ cases (99 patients). There was follow-up available on 94 patients and on $7.44 \%$ (7 patients) of them an unfavorable evolution was observed.

Group III risk factor incidence was $18.79 \%$ (53 patients). From 191 patients examined clinically on $71.2 \%$ (136 patients) a suspicion was formulated.

From 38 patients recorded with at least one hip IIa- type, on 29 follow-up data showed $6.89 \%$ (2 patients) treatment failure rate.

In 196 patients having at least one hip IIa+ type, follow-up was recorded on 114 patients. The unfavorable treatment rate was $8.77 \%$ (10 patients).

Dysplastic hips IIb type was identified in 55 patients with follow-up on $27.22 .22 \%$ (6 patients) presented unfavorable evolution on treatment.

\section{Discussion}

Among the criticisms leveled by opponents of the Graf method, late detected cases that initially were considered healthy on ultrasound examination, is probably the most difficult accusation $[7,8,10]$.

The purpose of this study is to identify two possible causes and to propose a solution for resolving them.

According to Graf, the inter-observer variation allowance is $2^{\circ}$, therefore we considered borderline hips those type I hips within this limit of $2^{\circ}\left(\alpha\right.$ angle: $60^{\circ}$ and $61^{\circ}$ ). Those hips could become type II if another examiner performs the ultrasound ( $\alpha$ angle: $58^{\circ}$ or $59^{\circ}$ ).

The asymmetry finding between the two hips is based on the same principle of interobserver variation of $2^{\circ}$ for each hip. Therefore, a difference of more than 4 degrees could be considered having a pathological substrate.

Analyzing the incidence of the risk factors in Group II and III, comparing with Group I, there is no significant difference. For Group I vs. Group II the Z-Score is 0.929 . The $p$-value is 0.17619 . The result is not significant $p$ being greater than 0.05 . For Group I vs. Group III the Z-Score is 0.1796 . The $p$-value is 0.85716 , the result being not significant for $\mathrm{p}$ value greater than 0.05 . No significant difference was 
Table I. p-values obtained when comparing the incidence of DDH risk factors and DDH clinical suspicion in the three groups.

\begin{tabular}{|l|l|l|l|}
\hline & Group I vs. Group II & Group I vs. Group III & Group II vs. Group III \\
\hline Risk factors & 0.17619 & 0.85716 & 0.5485 \\
\hline Clinical suspicion & 0.00027 & 0 & 0.00076 \\
\hline
\end{tabular}

Table II. p-values obtained when comparing the incidence of unsuccessful treatment rate of Group II vs. subgroups from Group III.

\begin{tabular}{|l|l|l|l|}
\hline & Group III, IIa- & Group III, IIa+ & Group III, IIb \\
\hline Group II & 0.46017 & 0.36317 & 0.01426 \\
\hline
\end{tabular}

observed when comparing de incidence of the risk factors in Group II vs. Group III. The Z-Score is -0.5994 . The p-value is 0.5485 . The result is not significant $\mathrm{p}$ being greater than 0.05 .

There were no significant differences between the three groups regarding the role of the risk factors in DDH pathogenesis (Table I).

Comparing pathological suspicion based on the clinical examination in Group I vs. Group II the Z-Score is -3.4599 . The $\mathrm{p}$-value is 0.00027 . The result is significant at $\mathrm{p}<0.05$. Similar significance was proofed comparing Group I vs. Group III; the Z-Score is -7.6455 . The p-value is 0 . The result is significant at $\mathrm{p}<0.05$. Comparing de incidence of pathological suspicion in Group II vs. Group III, also, significant difference was observed: the Z-Score is -3.1744 . The p-value is 0.00076 . The result is significant at $\mathrm{p}<0.05$. Those data reveal a high suspicion rate after the clinical examination, in groups II and III, comparing with the healthy population. That means that from this point of view, Group II might be considered heaving at least a dysplastic prognostic (Table I).

Regarding the unsuccessful treatment rate, Group II was compared with IIa-, IIa+ and IIb subgroups from Group III. In the first two situations, there is no significance between treatment of borderline and asymmetric hips and immature ones: Z-Score $=0.0995$, $p$-value $=0.46017$, respectively $\mathrm{Z}-\mathrm{Score}=-0.3472$. $\mathrm{p}$-value $=0.36317$; the result is not significant, $\mathrm{p}$ being greater than 0.05 .

But, comparing unsuccessful treatment rate from Group II with subgroup IIb, true DDH hips, a significant difference is observed: $Z-S c o r e=-2.1852$, $p$-value $=0.01426$, the result being significant at $\mathrm{p}<0.05$. Data reveals an increased correlation in the therapeutic results between Group II patients and those from mild delayed maturation subgroups from Group III (IIa-, IIa+) (Table II).

This study supports the idea of using ultrasound Graf method for diagnosing and monitoring the evolution of DDH.

The management of borderline and asymmetric hips should be performed in collaboration with an orthopedic surgeon. Even if the subjects in one of those situations are not considered having DDH, abductor attitude should be considered as a preventive method for a possible unfavorable evolution. We suggest that a minimal therapeutic intervention in borderline and asymmetric hips might lead to a decrease in the late detected cases number. Further studies on larger scale are required to validate these observations.

The study presents a few limitations. Because a single examiner conducted the ultrasound study, the interoperability variability could not be analyzed. The study is longitudinal retrospective and so the examiner was in the learning curve during the study period so that intraoperative variability cannot be analyzed. The therapeutic approach to the abnormal hip changes over time and tends to give up some old devices such Pavlik harness. The treatment has been instituted by several pediatric orthopedists in the absence of a standardized treatment protocol. There is no way to analyze the parent's compliance with the recommended therapy.

\section{Conclusion}

The study main finding could mean that borderline and asymmetric hips should be managed similarly to immature and potential dysplastic hips (IIa+ and IIa-), showing a potential pathological condition.

\section{References}

1. Heeres RH, Witbreuk MM, van der Sluijs JA. Diagnosis and treatment of developmental dysplasia of the hip in the Netherlands: national questionnaire of paediatric orthopaedic surgeons on current practice in children less than 1 year old. J Child Orthop. 2011;5:267-271.

2. Randall TL, Elaine NS. The Epidemiology and Demographics of Hip Dysplasia. ISRN Orthopedics 2011, doi: 10.5402/2011/238607. 3. Roposch A, Liu LQ, Hefti F, Clarke NM, Wedge JH. Standardized diagnostic criteria for developmental dysplasia of the hip in early infancy. Clin Orthop Relat Res. 2011;469:3451-3461.

4. Shorter D, Hong T, Osborn DA. Cochrane Review: Screening programmes for developmental dysplasia of the hip in newborn infants. Evid Based Child Health. 2013;8(1):11-54.

5. Laborie LB, Markestad TJ, Davidsen H, Brurås KR, Aukland SM, Bjørlykke JA, et al. Selective ultrasound screening for developmental hip dysplasia: effect on management and late detected cases. A prospective survey during 1991-2006. Pediatr Radiol. 2013;44(4):410-424.

6. Güler O, Şeker A, Mutlu S, Çerçİ MH, Kömür B, Mahİroğulları M. Results of a universal ultrasonographic hip screening program at a single institution. Acta Orthop Traumatol Turc. 2016;50(1):42-48. 7. Kyung BS, Lee SH, Jeong WK, Park SY. Disparity between Clinical and Ultrasound Examinations in Neonatal Hip Screening. 


\section{Medical Imaging}

Clin Orthop Surg. 2016;8:203-209.

8. Utzschneider S, Chita C, Paulus AC, Guenther C, Jansson V, Heimkes B. Discrepancy between sonographic and radiographic values after ultrasound-monitored treatment of developmental dysplasia of the hip. Arch Med Sci. 2016;12:1:145-149.

9. Ali AHA, Al Zahrani J, Elsayed AEA, Serhan OO. Role of
Ultrasound in Evaluation of Developmental Dysplasia of the Hip in Infants. Open Journal of Pediatrics 2017;7:1-12.

10. Bracken J, Tran T, Ditchfield M. Developmental dysplasia of the hip: controversies and current concepts. J Paediatr Child Health. 2012;48:963-973. 